\title{
Die Begründung der gesetzgeberischen Einschätzungsspielräume aus den Grenzen der verfassungsgerichtlichen Rechtsanwendung
}

Während einerseits die Befugnis des BVerfG zur Tatsachenfeststellung bei der Überprüfung eines Gesetzes unbestritten ist, wird andererseits allgemein angenommen, dass die verfassungsgerichtliche Tatsachenfeststellung gesetzgeberische Einschätzungsspielräume ${ }^{1}$ einzuräumen hat, die um so größer anzuerkennen sind, je unsicherer die in Rede stehenden generellen Tatsachen (sog. legislative facts) ${ }^{2}$ erscheinen. So hat etwa Brun-Otto Bryde ausgeführt: „Vor allem das Verhältnismäßigkeitsprinzip hindert jede Überlegung, das Verfassungsgericht von Tatsachenfeststellungen auszuschließen, fordert andererseits zwingend eine Zurückhaltung des Gerichts bei Tatsachen- und Prognosebewertungen, wenn der demokratische Gesetzgeber die Entscheidungsprärogative behalten soll, die ihm die grundgesetzliche Ordnung einräumt. " ${ }^{3}$ Fraglich ist allerdings, in welchem Sinne und inwieweit das Grundgesetz auf die gesetzgeberischen Einschätzungsspielräume hinweist. Die herrschende Meinung geht davon aus, die grundgesetzliche Ordnung als eine demokratische Ordnung zu verstehen, wonach der Gesetzgeber wegen seiner demokratischen Bedeutung eine zentrale Stellung besitzt. Im Falle empirischer Unsicherheit hat er die Prärogative, auch wenn hinsichtlich der Erforschung von generellen Tatsachen die Kompetenz sowohl des Gesetzgebers als auch des Verfas-

1 Nach Marius Raabe lässt sich der Begriff des Einschätzungsspielraums des Gesetzgebers wie folgt bestimmen: „Der Einschätzungsspielraum ist die Kompetenz des Gesetzgebers, beim Erlass eines Gesetzes einen unsicheren empirischen Satz als bei der Grundrechtsprüfung des Gesetzgebers zugrunde zu legende empirische Prämisse festzusetzen. “M. Raabe, Grundrechte und Erkenntnis. Der Einschätzungsspielraum des Gesetzgebers, 1998, S. 53.

2 Zum Begriff der generellen Tatsachen bzw. der legislative facts, mit denen sich das BVerfG bei der Normenkontrolle und der Gesetzesverfassungsbeschwerde befasst, vgl. K. J. Philippi, Tatsachenfeststellungen des BVerfG: Ein Beitrag zur rational-empirischen Fundierung verfassungsgerichtlicher Entscheidungen, 1971, S. 6-9; F. Ossenbühl, Die Kontrolle von Tatsachenfeststellungen und Prognoseentscheidungen durch das Bundesverfassungsgericht, in: C. Starck (Hrsg.), Bundesverfassungsgericht und Grundgesetz, Bd. I, 1976, S. 458 ff., 464-466; W. Kluth, Beweiserhebung und Beweiswürdigung durch das Bundesverfassungsgericht, NJW 1999, S. 3513 ff., 3514-3516; O. Lepsius, Sozialwissenschaften im Verfassungsrecht - Amerika als Vorbild?, JZ 2005, S. 1 ff., 1; zu legislative facts aus amerikanischer Diskussion etwa: $K$. C. Davis, An Approach to Problems of Evidence in the Administrative Process, 55 Harv. L. Rev. 364, 402-416 (1942); A. Hoffman, Corralling Constitutional Fact: De Novo Fact Review in the Federal Appellate Courts, 50 Duke L. J. 1427, 1434-1435 (2001); M. S. Paulsen, Abrogating Stare Decisis by Statute: May Congress Remove the Precedential Effect of Roe and Casey?, 109 Yale L. J. 1535, 1562-1563 (2000). Da die vorliegende Arbeit sich auf das Verhältnis zwischen Verfassungsgericht und Gesetzgeber konzentriert, wobei in erster Linie nicht konkrete Tatsachen, sondern vielmehr generelle sozio-ökonomische Daten interessieren (dazu wiederum Philippi, ebenda, S. 10-12), beziehen sich Tatsachen hier nur auf die generellen Tatsachen. Zur Feststellung konkreter Einzeltatsachen vgl. etwa Kluth, ebenda, S. 3515.

3 B.-O. Bryde, Tatsachenfeststellungen und soziale Wirklichkeit in der Rechtsprechung des Bundesverfassungsgerichts, in: P. Badura/H. Dreier (Hrsg.), FS 50 Jahre Bundesverfassungsgericht, Band I, 2001, S. 533 ff., 555. 
sungsgerichts anzuerkennen ist. ${ }^{4}$ Aus dieser Perspektive liegt es nahe, dass sich die Einschätzungsspielräume des Gesetzgebers gerade aus einer Kompetenzüberlegung ergeben, die großes Gewicht auf Demokratie legt: Die Reichweite des gesetzgeberischen Einschätzungsspielraums solle davon abhängen, inwieweit das BVerfG die demokratische Dignität des Gesetzgebers zu respektieren habe.

Die Einräumung der Einschätzungsspielräume lässt sich zwar als Folge des spannenden Verhältnisses zwischen Verfassungsgerichtsbarkeit und demokratischer Gesetzgebung ansehen; ungeklärt bleibt jedoch, worin die Grenzen zwischen den beiden liegen sollen. Wenn die Verfassungsgerichtsbarkeit unter dem Grundgesetz der Demokratie nicht entgegensteht, sondern sie durch normative Kontrolle gewährleistet, ${ }^{5}$ so scheint die Forderung unplausibel zu sein, das BVerfG solle sich schon aus demokratischem Grund gegenüber dem Gesetzgeber zurückhalten. Dies besagt freilich nicht, dass nach dem Grundgesetz, das ein demokratische gesetzgebende Gewalt kontrollierendes Verfassungsgericht einrichtet, die Einschätzungsspielräume des Gesetzgebers gar nicht existieren oder existieren sollen. Im Gegenteil soll nicht verleugnet werden, dass das Verfassungsgericht auf Schwierigkeiten stoßen muss, indem es einerseits als Rechtsanwendungsorgan die Tatsachen zu konfrontieren und festzustellen hat, andererseits aber gerade angesichts seiner Rolle als Rechtsanwendungsorgan nicht in der Lage ist, empirische Themen sachverständig zu behandeln. In dieser Hinsicht spielt zur verfassungsrechtlichen Begründung der Einschätzungsspielräume des Gesetzgebers nicht das Demokratieprinzip, sondern vielmehr die Grenze der verfassungsgerichtlichen Rechtsanwendung, die sich aus den Grenzen des normativen Sollens gegenüber dem faktischen Sein ergibt, die entscheidende Rolle. Im Folgenden soll zunächst gezeigt werden, inwiefern die allgemeine Ansicht, die oben dargelegt worden ist, den Schwerpunkt zur Begründung der Einschätzungsspielräume des Gesetzgebers verkannt hat. Sodann ist nachzugehen, auf welcher Grundlage und in welcher Weise sich die Grenzen zwischen gesetzgeberischen Einschätzungsspielräumen und verfassungsgerichtlicher Kontrolle im Wege verfassungsgerichtlicher Tatsachenfeststellung bilden sollen.

\section{Die Einschätzungsspielräume des Gesetzgebers aus kompetenzrechtlichen Erwägungen}

\section{Die verfassungsgerichtliche Einräumung der Einschätzungsspielräume des Gesetzgebers durch unterschiedliche Kontrolldichte als Respekt vor der Demokratie}

Mit der Verstärkung der Verfassungsgerichtsbarkeit im Gefüge der Staatsfunktionen wird allgemein angenommen, dass das BVerfG seine Kompetenz überschreiten und in die demokratische gesetzgebende Gewalt eingreifen kann, indem es die Aufgabe des

4 Daher wird die Tatsachenfeststellung häufig als ein Kompetenzproblem erfasst, das im Rahmen des Verhältnisses von Verfassungsgericht und Gesetzgeber vom Gegensatz zwischen Verfassungsgerichtsbarkeit und Demokratie ausgeht. So etwa Bryde (Fn. 3), S. 540, $553 \mathrm{ff}$.

5 Diese Auffassung vertritt schon H. Kelsen, Vom Wesen und Wert der Demokratie (1929), 2. Aufl. 1963, S. 75 f.; ders., Wesen und Entwicklung der Staatsgerichtsbarkeit, in: VVDStRL 5 (1929), S. 2 ff., 81. 
Grundrechtsschutzes erfüllt. ${ }^{6}$ Infolgedessen konzentrieren sich die heutigen Untersuchungen überwiegend darauf, Verfassungsgerichtsbarkeit gegenüber demokratischer Gesetzgebung abzugrenzen. In dieser Hinsicht tauchen Grundrechte und Demokratie als gegenüberzustellende Begriffe auf: Während das BVerfG auf der Seite von Grundrechten steht, ist der Gesetzgeber durch seine demokratische Bedeutung gekennzeichnet. Daher ist das spannende Verhältnis zwischen Verfassungsgericht und Gesetzgeber überwiegend als die Spannung zwischen Grundrechten und Demokratie verstanden. ,Je weiter also die Grundrechte im Ergebnis reichen, desto weniger Möglichkeiten bleiben dem Gesetzgeber. Umgekehrt gilt: Je mehr der Gesetzgeber entscheiden darf, desto kleiner ist der grundrechtliche Bereich, der als definitiv mehrheitsfest geschützt verbleibt. "7 Gerade im Hinblick auf die Spannung über die Reichweite der Grundrechte auf der einen Seite und der Demokratie auf der anderen Seite wird heutzutage vielfach gefordert, dem Gesetzgeber bestimmte Spielräume zu sichern, innerhalb denen die demokratische Gesetzgebung keiner verfassungsgerichtlichen Kontrolle unterliegt. ${ }^{8}$ Die Frage lautet also: Unter welcher Voraussetzung und in welchem Umfang soll das BVerfG auf seine Kontrolle verzichten, um die gesetzgeberischen Spielräume zu garantieren und dadurch seinen Respekt vor der Demokratie darzustellen?

Diese Frage hat das BVerfG im Mitbestimmungsurteil aus dem Jahre $19799^{9}$ systematisch beantwortet. Das Gericht ging davon aus, dass die gesetzgeberischen Spielräume dort entstehen, wo faktische Unsicherheit, vor allem aber Unsicherheit über die Zukunft, vorliegt. Dementsprechend sind die tatsachenbezogenen Einschätzungsspielräume des Gesetzgebers einzuräumen, auch wenn dem BVerfG nach § 26 Abs. 1 Satz 1 BVerfGG die Tatsachenerforschung und -feststellung zusteht. Infolgedessen setzt die verfassungsgerichtliche Befugnis zur Tatsachenfeststellung die Garantie der gesetzgeberischen Einschätzungsspielräume voraus, deren Reichweite im einzelnen unterschiedlich zu bestimmen ist: „Im einzelnen hängt die Einschätzungsprärogative des Gesetzgebers von Faktoren verschiedener Art ab, im besonderen von der Eigenart des in Rede stehenden Sachbereichs, den Möglichkeiten, sich ein hinreichend sicheres Urteil zu bil-

6 Zur Kritik an der Verfassungsgerichtsbarkeit aus dieser Sicht vgl. statt vieler E.-W. Böckenförde, Grundrechte als Grundsatznormen. Zur gegenwärtigen Lage der Grundrechtsdogmatik, Der Staat 20 (1990), S. 1 ff.; ders., Zur Lage der Grundrechtsdogmatik nach 40 Jahren Grundgesetz, 1989; W. Knies, Auf dem Weg in den „verfassungsgerichtlichen Jurisdiktionsstaat“?, in: FS K. Stern, 1997, S. 1155 ff., 1178 ff.; O. Höffe, Wieviel Politik ist dem Verfassungsgericht erlaubt?, Der Staat 38 (1999), S. 171 ff., 184 ff.; W. Brohm, Die Funktion des BVerfG - Oligarchie in der Demokratie?, NJW 2001, S. 1 ff.

7 So Raabe (Fn. 1) S. 15. Vgl. auch Kluth (Fn. 2), S. 3516: „Das BVerfG sieht sich also zwischen zwei Vorgaben: der Verpflichtung zum effektiven Grundrechtsschutz auch gegenüber dem Gesetzgeber einerseits und dem Respekt vor der demokratischen Dignität des unmittelbar vom Volk gewählten Parlaments andererseits.“.

8 Der sog. funktionell-rechtliche Ansatz lässt sich in dieser Hinsicht als Produkt der Überlegung ansehen, wonach etwa zwischen Handlungs- und Kontrollnormen zu unterscheiden ist. Vgl. dazu B.-O. Bryde, Verfassungsentwicklung: Stabilität und Dynamik im Verfassungsrecht der Bundesrepublik Deutschland, 1982, S. 306 f.: „Will man an der umfassenden Bindung aller staatlichen Gewalt an eine Verfassung mit universalem normativem Anspruch festhalten und trotzdem eine justizstaatliche Verdrängung der demokratischen und gewaltenteilenden Funktionenordnung vermeiden, muss man die Bedeutung der Verfassung als Handlungsmaßstab für die Staatsorgane und als Kontrollmaßstab für das BVerfG unterscheiden.“.

9 BVerfGE 50, 290. 
den, und der Bedeutung der auf dem Spiele stehenden Rechtsgüter. Demgemäß hat die Rechtsprechung des Bundesverfassungsgerichts, wenn auch im Zusammenhang mit anderen Fragestellungen, bei der Beurteilung von Prognosen des Gesetzgebers differenzierte Maßstäbe zugrunde gelegt, die von einer Evidenzkontrolle über eine Vertretbarkeitskontrolle bis hin zu einer intensivierten inhaltlichen Kontrolle reichen."10 Daher lässt sich der Aufbau der abgestuften Kontrolldichten als die Folge der verfassungsgerichtlichen (abgestuften) Anerkennung der gesetzgeberischen Einschätzungsspielräume ansehen, deren Reichweite von der Eigenart des in Rede stehenden Sachbereichs, den Möglichkeiten, sich ein hinreichend sicheres Urteil zu bilden, und der Bedeutung der auf dem Spiele stehenden Rechtsgüter abhängt.

Beachtenswert ist dabei, in welchem Sinne diese Aussage vom BVerfG ihre Bedeutung und Auswirkung hat. Die Entwicklung der Bundesverfassungsgerichtsentscheidungen nach dem Mitbestimmungsurteil zeigt, dass sich das BVerfG bei der Bestimmung seiner Kontrolldichte überwiegend auf die Bedeutung der Grundrechte konzentriert hat. ${ }^{11}$ Die Folge also: „Die Anforderungen an die Sicherheit der Prognose und Verlässlichkeit der Tatsachenbasis steigen lediglich mit der Bedeutung des Grundrechts und der Eingriffsintensität" ${ }^{12}$ Trifft diese Beobachtung zu, so lässt sich daraus ablesen, dass der Versuch des BVerfG, seine grundrechtsschützende Kontrollkompetenz gegenüber der demokratischen Gesetzgebung abzugrenzen, gerade der oben dargelegten Betrachtungsweise zum Spannungsverhältnis zwischen Verfassungsgericht und Gesetzgeber entspricht. Die Einschätzungsspielräume des Gesetzgebers müssen demgemäß mit der Schwächung der Bedeutung der auf dem Spiele stehenden Grundrechte und der Eingriffsintensität erweitert werden. Dagegen scheint relativ unklar, unter welchen Voraussetzungen die anderen Faktoren, die nach dem Mitbestimmungsurteil im Rahmen der Bestimmung der Reichweite gesetzgeberischer Einschätzungsspielräume auch eine Rolle spielen sollen, geltendzumachen sind. ${ }^{13}$

Spiegelt die Praxis des BVerfG einerseits die Auffassung genau wider, die Spannung zwischen Verfassungsgerichtsbarkeit und Gesetzgebung stelle sich als die Spannung

10 BVerfGE 50, 290 (332f.).

11 Vgl. K. Schlaich/S. Korioth, Das Bundesverfassungsgericht - Stellung, Verfahren, Entscheidungen, 6. Aufl. 2004, Rn. 537. Dort wird behauptet, die verschiedenen Kontrolldichten seien nicht ,funktionell-rechtliche Kompetenzkritierien selbständiger Art“, sondern vielmehr ,materiell-rechtlich gesteuert“. Ähnlich W. Heun, Funktionell-rechtliche Schranken der Verfassungsgerichtsbarkeit, 1992, S. 37-40; C. Simons, Grundrechte und Gestaltungsspielraum. Eine rechtsvergleichende Untersuchung zum Prüfungsinstrumentarium von Bundesverfassungsgericht und US-amerikanischem Supreme Court bei der Normenkontrolle, 1999, S. 228-233; K. Chryssogonos, Verfassungsgerichtsbarkeit und Gesetzgebung. Zur Methode der Verfassungsinterpretation bei der Normenkontrolle, 1987, S. 190. Vgl. auch S.-P. Hwang, Verfassungsgerichtlicher Jurisdiktionsstaat? Eine rechtsvergleichende Analyse zur Kompetenzabgrenzung von Verfassungsgericht und Gesetzgeber in den USA und der Bundesrepublik Deutschland, 2005, S. 180-184.

12 Heun (Fn. 11), S. 38.

13 Im Hinblick auf den viel bemerkten Gegensatz zwischen Grundrechten und Demokratie wird auch bei der Diskussion um den Faktor der Eigenart des betroffenen Sachbereiches die funktionell-rechtliche Eigenschaft bzw. die demokratische Bedeutung des Gesetzgebers herausgestellt. Vgl. z.B. G. F. Schuppert, Self-restraints der Rechtsprechung: Überlegung zur Kontrolldichte in der Verfassungs- und Verwaltungsgerichtsbarkeit, DVBl 1988, S. 1191 ff., $1192 \mathrm{f}$. 
zwischen Grundrechten und Demokratie dar, so reflektiert sie andererseits, dass das Thema zu den gesetzgeberischen Einschätzungsspielräumen, die nach dem Mitbestimmungsurteil nicht lediglich von der Bedeutung der Grundrechte und der Eingriffsintensität abhängen sollen, noch nicht umfassend behandelt worden ist. Die Überbetonung des Konfliktverhältnisses von Grundrechten und Demokratie würde dazu führen, dass der demokratische Gesetzgeber jedenfalls mit seinen Einschätzungsspielräumen Vorrang vor dem Verfassungsgericht hätte, solange kein schwerer Grundrechtseingriff vorliegt, der erst die verfassungsgerichtliche Kontrolle rechtfertigt. In der Tat aber stehen nach dem Grundgesetz Grundrechte und Demokratie nicht in allen Zusammenhängen entgegen, so dass die Abgrenzung zwischen Verfassungsgerichtsbarkeit und Gesetzgebung nicht einfach durch den Ausgleich zwischen Grundrechtsschutz und Demokratiegewährleistung thematisiert werden kann. So gesehen greift die Ansicht, die Einräumung der Einschätzungsspielräume des Gesetzgebers lasse sich bereits und zwar ausschließlich auf demokratischer Grundlage rechtfertigen, zu kurz.

\section{Verfassungsgerichtsbarkeit im Grundgesetz: Das BVerfG als Hüter der demokratischen Verfassung}

Sowohl in rechtsgeschichtlicher als auch in rechtsvergleichender Hinsicht zeichnet sich das Grundgesetz in erster Linie durch die Einrichtung einer machtvollen Verfassungsgerichtsbarkeit aus, die sich nicht von der Politik abtrennt, sondern sie kontrolliert. ${ }^{14}$ Vor diesem Hintergrund spiegelt das Grundgesetz einen Paradigmenwechsel in der Recht-Politik-Problematik wider: Im Rahmen der Verfassungsgerichtsbarkeit müssen die rechtsfreien Räume der Politik erheblich eingeschränkt werden, so dass der Gesetzgeber immer wieder der verfassungsgerichtlichen Kontrolle unterliegt. ${ }^{15}$ Auf der anderen Seite aber ist nicht zu verleugnen, dass die Rolle der gesetzgebenden Gewalt im Gefüge der heutigen Staatsfunktionen nicht nur aus institutioneller Perspektive bzw. auf der Grundlage des Grundgesetzes begriffen, sondern auf ihre Bedeutung für die ganze Rechtsordnung im Lichte der deutschen Rechtstradition zurückgeführt werden muss. In dieser Hinsicht wird einerseits der Gesetzgeber stark gebunden; andererseits spielt er aber aufgrund seiner zentralen Stellung sowohl im rechtsstaatlichen als auch im demo-

14 Vgl. G. Roellecke, Aufgaben und Stellung des Bundesverfassungsgerichts im Verfassungsgefüge, in: J. Isensee/P. Kirchhof (Hrsg.), HStR II, 1987, § 53, S. 191 f.; auch U. Scheuner, Verfassungsgerichtsbarkeit und Gesetzgebung, DÖV 1980, S. 473 ff. 474; F. Ossenbühl, Aktuelle Probleme der Gewaltenteilung, DÖV 1980, S. 545 ff., 547 f. Daher wird behauptet, die Sorge um die Grenzen der Verfassungsgerichtsbarkeit aus Sicht des traditionellen Gewaltenteilungsprinzips werde „Zweitrangig“ und sogar „obsolet“, denn „die Grenzen des BVerfG sind ... mit den von der Verfassung gesteckten Grenzen identisch." So Simons (Fn. 11), S. 18. Gerade in diesem institutionellen Sinne unterscheidet sich die deutsche Verfassungsgerichtsbarkeit grundlegend von der US-amerikanischen. Vg. dazu Hwang (Fn. 11), S. 17-22.

15 Dies spiegelt sich vor allem dadurch wider, dass die Verfassungsgerichtsbarkeit die unmittelbare Bindungswirkung der Grundrechte (Art. 1 III und Art. 20 III GG) geltend macht. 
kratischen Kontext stets eine schwerwiegende Rolle. ${ }^{16}$ Infolgedessen wird die sog. politische Gestaltungsfreiheit des Gesetzgebers immer wieder behauptet und herausgestellt, die sich als die Darstellung dieser zweiseitigen Betrachtung ansehen lässt.

Indem die gesetzgeberische Gestaltungsfreiheit sich aus der Überlegung ergibt, der Gesetzgeber solle vor allem in rechtsgeschichtlicher Hinsicht trotz der Einrichtung der Verfassungsgerichtsbarkeit eine zentrale Stellung im heutigen demokratischen Rechtsstaat besitzen, kann jedoch die demokratische Dignität des Gesetzgebers überschätzt werden, wobei die demokratische Bedeutung der Verfassungsgerichtsbarkeit auch relativ vernachlässigt wird. Zwar liegt der grundgesetzlichen Ordnung das Demokratieprinzip zugrunde; allerdings beabsichtigt das Grundgesetz nicht, Demokratie ausschließlich durch den Gesetzgeber zu verwirklichen und zu gewährleisten. Umgekehrt muss der Gesetzgeber deshalb kontrolliert werden, weil er ansonsten seine Gewalt missbrauchen und dadurch die Demokratie beeinträchtigen kann. ${ }^{17}$ So gesehen stehen Verfassungsgerichtsbarkeit und Demokratie trotz und auch wegen der verfassungsgerichtlichen Befugnis zur Kontrolle der demokratischen Gesetzgebung nicht entgegen,

16 Vgl. die abweichende Meinung der Richterinnen Osterloh und Lübbe-Wolff und des Richters Gerhardt zum Urteil des Zweiten Senats vom 27. Juli 2004, in: BVerfGE 111, 226 (274 ff.), 274: „Im Rechtsstaat des Grundgesetzes ist die Gesetzgebung das wichtigste Mittel politischer Gestaltung." Zur zentralen Rolle des Gesetzgebers besonders in seinem Verhältnis zur Verwaltung vgl. etwa D. Jesch, Gesetz und Verwaltung. Eine Problemstudie zum Wandel des Gesetzmäßigkeitsprinzips, 1961, S. 92 ff.; P. Badura, Die parlamentarische Volksvertretung und die Aufgabe der Gesetzgebung, ZG 1987, S. 300 ff.; O. Lepsius, Die erkenntnistheoretische Notwendigkeit des Parlamentarismus, in: M. Bertschi u.a. (Hrsg.), Demokratie und Freiheit, 1999, S. 123 ff., 124 f.; vgl. auch G. F. Schuppert (Hrsg.), Das Gesetz als zentrales Steuerungsinstrument des Rechtsstaates, 1998. Die zentrale Stellung der Legislative im Kontext der deutschen Rechtstradition geht an erster Stelle auf den Rechtsstaatsgedanken zurück, der, anders als die Rule of Law vor dem Hintergrund des Common Law, dadurch gekennzeichnet ist, dass nicht das Gericht, sondern der Gesetzgeber die führende Rolle zur Gewährleistung der individuellen Freiheit spielt. Zwar hat die Hinwendung des Grundgesetzes zum „materiellen“ Rechtsstaat nach 1945 wesentliche Auswirkungen auf den Status des Gesetzgebers (dazu E.-W. Böckenförde, Entstehung und Wandel des Rechtsstaatsbegriffs, in: ders., Recht, Staat, Freiheit, 1991, S. 143 ff., 164 ff.). Allerdings hält die grundgesetzliche Entscheidung für die parlamentarische Demokratie immerhin daran fest, dass , das zentrale Legitimations- und Entscheidungsorgan der Demokratie das Parlament ist." (so die Formulierung bei $P$. Kirchhof, Die Zukunft der Demokratie im Verfassungsstaat, JZ 2004, S. 981 ff., 984). Folgerichtig wird im Rahmen des Grundgesetzes der Gesetzgeber auf Grund des Demokratieprinzips immer wieder in den Vordergrund gerückt. So gesehen besitzt der Gesetzgeber im Hinblick auf seine führende Rolle in der Entwicklung der deutschen Rechtsgeschichte nach wie vor eine zentrale Bedeutung.

17 Vor dem Demokratiemissbrauch warnt etwa W. Kägi, Rechtsstaat und Demokratie (Antinomie und Synthese), in: Festgabe Z. Giacometti, 1953, S. 107 ff., 188 ff. 
sondern sie unterstützen einander. ${ }^{18}$ Weiterhin ist im Hinblick auf die Funktion des Verfassungsgerichts in der - im allgemeinen angenommenen - pluralistischen Demokratie, ${ }^{19}$ die Minderheitsrechte durch die Gewährleistung steter Kompromissmöglichkeiten zwischen Majorität und Minorität zu schützen, ferner anzuerkennen, dass selbst die Verfassungsgerichtsbarkeit eine demokratische Bedeutung enthält. ${ }^{20}$ Auf dieser Ebene verdeutlicht sich, dass die grundgesetzliche Demokratie sich nicht in der Legislative erschöpft, sondern vielmehr durch die sowohl kontrollierende als auch schützende Funktion der Verfassungsgerichtsbarkeit geltend zu machen ist. Demzufolge ist anzunehmen, dass das Demokratieprinzip nicht als der grundgesetzliche Maßstab dazu dienen kann, die Kompetenzen von Verfassungsgericht und Gesetzgeber klar abzugrenzen, weil das Verfassungsgericht eben nach dem Demokratieprinzip die Aufgabe trägt, Demokratie durch die normative Kontrolle gesetzgeberischer Entscheidungen zu garantieren. In dem Sinne, dass das Grundgesetz keine Freiräume für den Gesetzgeber vorbestimmt, die aus demokratischem Grund voraussetzungslos und vorbehaltlos vor der verfassungsgerichtlichen Überprüfung geschützt werden müssen, soll das Demokratieprinzip nicht als die Grenze zwischen Verfassungsgerichtsbarkeit und Gesetzgebung begriffen werden.

Dem Vorhergehenden ist nicht zu entnehmen, dass unter dem Grundgesetz der Gesetzgeber gar keine Gestaltungsfreiheit genießen dürfte. Dass das Demokratieprinzip nicht als die Grenze für das BVerfG gelten kann, besagt nur, dass dieses im Hinblick auf die kontrollierende und damit unterstützende Bedeutung der Verfassungsgerichtsbarkeit für die Demokratie nicht in der Lage ist, zur Erklärung der Grenzziehung zwischen Verfassungsgerichtsbarkeit und Gesetzgebung beizutragen, nicht aber, dass nach dem Grundgesetz die verfassungsgerichtliche Kontrolle keine Spielräume für den Gesetzgeber überlässt. Vielmehr ist anzunehmen, dass das BVerfG mit dem Rechtsanwendungscharakter seine verfassungsrechtlichen Schranken finden muss. Bereits die Mitbestimmungsentscheidung erläuterte, dass die empirische Unsicherheit zur Zurückhaltung verfassungsgerichtlicher Kontrolle der gesetzgeberischen Entscheidung und deswegen auch zur Einschätzungsprärogative des Gesetzgebers führen würde. Offenkundig rückt diese Auffassung die empirischen Faktoren in den Vordergrund, die im Rahmen der Verfassungsgerichtsbarkeit als die Grenzen der verfassungsgerichtlichen

18 Vgl. dazu auch Kägi, ebenda, S. 134-138; D. Grimm, Verfassungsgerichtsbarkeit - Funktion und Funktionsgrenzen im demokratischen Staat, in: W. Hoffmann-Riem (Hrsg.), Sozialwissenschaften im Studium des Rechts, 1977, S. 83 ff., 89, 95 ff.; N. Geis, Autorität und Macht des Verfassungsgerichts, in: B. Rill (Hrsg.), Fünfzig Jahre freiheitlich-demokratischer Rechtsstaat. Vom Rechtsstaat zum Rechtswegestaat, 1999, S. 137 ff., 139. Zur Notwendigkeit einer rechtlichen Kontrolle politischen Handelns unter dem Grundgesetz vgl. etwa H. Laufer, Verfassungsgerichtsbarkeit und politischer Prozess. Studien zum Bundesverfassungsgericht der Bundesrepublik Deutschland, 1968, S. 45, 284 ff., 336 ff.; Scheuner (Fn.), S. 473 f.; R. Wahl, Elemente der Verfassungsstaatlichkeit, in: JuS 2001, S. $1041 \mathrm{ff} ., 1046$.

19 Zur pluralistischen Demokratie unter dem Grundgesetz vgl. etwa A. Hanebeck, Der demokratische Bundesstaat des Grundgesetzes, 2004, S. 84-86; P. Badura, Staatsrecht. Systematische Erläuterung des Grundgesetzes für die Bundesrepublik Deutschland, 3. Aufl. 2003, S. 274.

20 Dazu näher Kelsen, Vom Wesen und Wert der Demokratie (Fn. 5) S. 57 f.; ders., Wesen und Entwicklung der Staatsgerichtsbarkeit (Fn. 5), S. 81; ders., Allgemeine Staatslehre, 1925, S. 359 . 
Überprüfung dienen sollen. ${ }^{21}$ Gerade in dieser Hinsicht lohnt es sich, die verfassungsgerichtlichen Andeutungen aus dem Mitbestimmungsurteil aus einer über den Gegensatz zwischen Grundrechten und Demokratie hinausgehenden Perspektive wieder zu entdecken. Mit Blick auf die Darstellung vom BVerfG muß die Reichweite der gesetzgeberischen Spielräume nicht nur von der Bedeutung der Grundrechte, sondern auch von der Eigenart des in Rede stehenden Sachbereiches und den Möglichkeiten, sich ein hinreichend sicheres Urteil zu bilden, bestimmt werden. Im Hinblick auf die Bedeutung der empirischen Unsicherheit für die Schrankensetzung verfassungsgerichtlicher Kontrolle lässt sich die im Mitbestimmungsurteil hervorgehobene „Eigenart des betroffenen Sachbereiches“ als ein konkretisierter Maßstab zur Abgrenzung von Verfassungsgerichtsbarkeit und Gesetzgebung auffassen, der in erster Linie nicht das Demokratieprinzip, sondern vielmehr die empirische Ungewissheit als die Grenzen verfassungsgerichtlicher Überprüfung herausstellt. Infolgedessen stellt sich die Frage, weshalb und auf welche Weise die empirischen Faktoren bzw. Voraussetzungen zur Begrenzung der verfassungsgerichtlichen Kontrolle auf der einen Seite und zur Begründung und Gewährleistung der gesetzgeberischen Einschätzungsspielräume auf der anderen Seite ins Spiel kommen sollen.

\section{Verfassungsgerichtsbarkeit als tatsachenforschende Rechtsanwendung}

Als ein Rechtsanwendungsorgan muss das BVerfG im Rahmen der Kontrolle der gesetzgeberischen Entscheidung nicht nur an die Verfassungsnorm gebunden werden, sondern naturgemäß auch über die Verfassungsmäßigkeit des in Rede stehenden Gesetzes sachgerecht entscheiden. Zur Überprüfung des Gesetzes und damit zur Lösung des konkreten Streitfalles, d.h. zur Entscheidung über die Verfassungsmäßigkeit des Gesetzes, liegt es nahe, dass das BVerfG, im Schnittpunkt zwischen Verfassungssystematik und Sachgerechtigkeit stehend, alle generellen Tatsachen festzustellen hat, die im Rahmen des geprüften Gesetzes eine Rolle spielen und auch gerade aus diesem Grund notwendig für die verfassungsgerichtliche Rechtsanwendung sind. Etwa bei der Verhältnismäßigkeitsprüfung also steht die Tatsachenfeststellung des BVerfG im Mittelpunkt, indem geprüft werden muss, ob die getroffene Regelung zur Zweckerreichung „geeignet" und „erforderlich“ ist. ${ }^{22}$ Gerade die Notwendigkeit zur Tatsachenfeststellung hat die Auseinandersetzung des BVerfG mit dem Gesetzgeber in dem Sinne zugespitzt, als die Tatsachenforschung nicht nur der verfassungsgerichtlichen Rechtsanwendung, sondern selbstverständlich auch der gesetzgeberischen Rechtsetzung zugrunde liegt.

21 Gerade die verfassungsgerichtliche Gewichtlegung auf die empirischen Faktoren im Mitbestimmungsurteil aber hat Kritik bewirkt. Vgl. z.B. R. Schmidt, Das Mitbestimmungsgesetz auf dem verfassungsrechtlichen Prüfstand. Bemerkungen zum Mitbestimmungsurteil des BVerfG vom 1. März 1979 (BVerfGE 50. S. 290 ff.), Der Staat 19 (1980), S. 235 ff., 242: „Hierin liegt ein entscheidender Mangel, weil der topos ,Prognosespielraum“ nicht nur durch den jeweiligen Sachbereich, sondern vor allem durch die möglicherweise verletzten Grundrechte bestimmt werden muss.".

22 Zur Bedeutung der Tatsachenforschung bei der Verhältnismäßigkeitsprüfung vgl. etwa $R$. Alexy, Theorie der Grundrechte, 1985, S. 101 ff.; Raabe (Fn. 1), S. 332 f; P. Lerche, Übermaß und Verfassungsrecht. Bemerkungen zur Wiederauflage (1999), in: ders., Ausgewählte Abhandlungen, 2004, S. 244 ff., 248 f. 
Zwar ist nach den $§ \S 26-29$ BVerfGG das BVerfG zur tatsächlichen Beweiserhebung verpflichtet; als Rechtsanwendungsorgan aber darf es sich nicht als Ersatzgesetzgeber betätigen.

\section{Tatsachenfeststellung im Wege verfassungsgerichtlicher Rechtsanwendung}

Um die Grenzen der verfassungsgerichtlichen Tatsachenfeststellung herauszufinden, muss an erster Stelle festgelegt werden, worauf die Notwendigkeit zur Tatsachenfeststellung durch das BVerfG beruht. Wie dargelegt, ergibt sich die Befugnis des BVerfG zur Feststellung von generellen Tatsachen schon daraus, dass das BVerfG über die Verfassungsmäßigkeit eines Gesetzes zu entscheiden hat, dessen Entstehungshintergründe bzw. sozio-ökonomische Grundlagen infolgedessen zum „Teil des Sachverhalts“ für die verfassungsgerichtliche Rechtsanwendung werden, ,,auf den sich die richterliche Konkretisierungsaufgabe bezieht" . ${ }^{23}$ Dem ist zu entnehmen, dass die Tatsachenfeststellung des BVerfG, das „,keine Tatsacheninstanz sein will“, ${ }^{24}$ eng mit seiner Rechtsanwendungsaufgabe verbunden ist. Das BVerfG hat die generellen Tatsachen deshalb festzustellen, weil es die (Verfassungs-)norm auf den diese Tatsachen zugrunde legenden Einzelfall anzuwenden hat. ${ }^{25}$ So gesehen lässt sich sagen, dass im Rahmen der verfassungsgerichtlichen Notwendigkeit zur Rechtsanwendung die Begründung und die Begrenzung der verfassungsgerichtlichen Kompetenz zur Tatsachenfeststellung zusammenfallen. Nur am Maßstab der Verfassungsnorm kann sich die verfassungsgerichtliche Tatsachenfeststellung geltend machen und rechtfertigen. Zum Beispiel muss also das Gericht in der neueren Entscheidung zum Altenpflegegesetz einschlägige Tatsachen ermitteln und feststellen, um am Maßstab des Art. 72 Abs. 2 GG, wonach „dem Bundesgesetzgeber kein Beurteilungsspielraum belassen werden sollte“, ${ }^{26}$ die Verfassungsmäßigkeit des Gesetzes über die Berufe in der Altenpflege nach der „Erforderlichkeitsklausel" zu prüfen, die, nach der Auffassung des Gerichts, eine verfassungsgerichtliche Überprüfung der gesetzgeberischen Tatsachenerforschung ermöglicht und insofern die Tatsachenermittlung des Verfassungsgerichts rechtfertigt: „Die Konkretisierung der Bedürfnisklausel sollte der Justitiabilität des Art. 72 Abs. 2 GG dienen. Sie sollte eine andere rechtliche Qualität der darin enthaltenen Begriffe bewirken; die Kompetenzausübung durch den Bund sollte nicht mehr nur auf Ermessensmissbrauch überprüfbar sein und die Tatbestandsmerkmale sollten zu - zwar unbestimmten, aber - voll nachprüfbaren Gesetzesbegriffen werden, damit das Bundesverfassungsgericht die Voraussetzungen des Art. 72 Abs. 2 GG ebenso nachprüfen könne wie die grundgesetzlichen Bestimmungen anderer Kompetenzen. Verfassungsgerichtlicher Kontrolle sollte dabei

23 Bryde (Fn. 3) S. 533 f. Von den generellen Tatsachen dieser Art unterscheidet Bryde ,solche gesamtgesellschaftlichen sozio-ökonomischen Daten und Wirklichkeitsvorstellungen des Gerichts, die sich auf den gesellschaftlichen Kontext seiner Entscheidung beziehen und die Auslegung der Norm beeinflussen.".

24 Vgl. Lepsius (Fn. 2), S. 2.

25 Vgl. zur Rolle der Tatsachen im Rahmen der gerichtlichen Subsumtion auch G. Struck, Rechtswissenschaft und Soziologie, in: D. Grimm (Hrsg.), Rechtswissenschaft und Nachbarwissenschaften, Bd. 1, 1976, S. 13 ff., 24.

26 BVerfGE 106, 62 (142). 
auch der Maßstab der ,Erforderlichkeit‘ einer bundesgesetzlichen Regelung unterworfen sein." 27 Gerade dem lässt sich entnehmen, dass das BVerfG seine Befugnis zur Tatsachenfeststellung gerade auf dem Boden begründet, dass es aufgrund der Erforderlichkeitsklausel des Art. 72 Abs. 2 GG zur Überprüfung der Tatsachenermittlung des Gesetzgebers verpflichtet ist. Daraus folgt ferner, dass die Tatsachenfeststellung des BVerfG, deren Reichweite auf die anzuwendende Verfassungsnorm zurückzuführen ist, nicht zwangsläufig dazu führen würde, die Tatsachenfeststellungsbefugnis des Gesetzgebers in Kauf zu nehmen, denn die beiden haben im Wesentlichen unterschiedliche Richtungen und Reichweiten. Während der Gesetzgeber aus der Notwendigkeit zur Schaffung einer Rechtsnorm die Tatsachen zu erforschen hat, beruht die Tatsachenermittlung des BVerfG auf seiner Notwendigkeit zur Überprüfung des Gesetzes durch die Anwendung der vorgegebenen Verfassungsnorm. Bereits an dieser Stelle zeigt sich deutlich, dass die Tatsachenermittlung des BVerfG rechtliche Vorgaben voraussetzen muss, während diejenige des Gesetzgebers relativ offen, d.h. wenig rechtsgebunden ist.

Wie weitgehend anerkannt, entsteht dieser Unterschied aus der methodologischen Gegenüberstellung und Abgrenzung von Rechtsanwendung und Rechtsetzung. Während die gerichtliche Rechtsanwendung durch Rechtsbindung, durch Individualisierungsleistung und durch Retrospektivität gekennzeichnet ist, zeichnet sich die gesetzgeberische Rechtsetzung durch ihre Offenheit, Allgemeinheit sowie Zukunftsbezogenheit aus. ${ }^{28}$ Daraus folgt, dass die Tatsachen im Rahmen der Verfassungsgerichtsbarkeit und der Gesetzgebung unterschiedlich zu behandeln sind, und dass infolgedessen die Begrenzung der verfassungsgerichtlichen Befugnis zur Tatsachenfeststellung auf der einen Seite und die Einräumung der Einschätzungsspielräume des Gesetzgebers auf der anderen Seite letztlich auf die Reichweite der Rechtsanwendung gegenüber der Rechtsetzung zurückgehen müssen. Die Folge also: Dient die Tatsachenforschung als Komponente bzw. Ausgangspunkt einer Gesetzgebung, die selbstinitiativ und in ihrer Entstehung wenig verrechtlicht ist, ${ }^{29}$ so hat sie ihrer Funktion nach nicht von den normativen, sondern vielmehr von den faktischen Voraussetzungen auszugehen, die sich entweder auf gesicherte Tatsachen oder auf ungewisse Zukunft beziehen können. Bereits dies deutet an, aus welchem Grund die zukunftsbezogene empirische Unsicherheit im Rahmen der Einräumung gesetzgeberischer Einschätzungsspielräume eine Rolle spielt. ${ }^{30}$ Wird im Gegenteil die Tatsachenfeststellung als Bestandteil der verfassungsgerichtlichen Rechtsanwendung angesehen und vorgenommen, die in dem Sinne rechtlich determiniert ist, dass sich das Gericht in seiner Entscheidungsrechtfertigung nur auf Recht beziehen darf, ${ }^{31}$ so steht fest, dass die Reichweite dieser Tatsachenfeststellung von der Reichweite der vorgegebenen Verfassungsnorm zu bestimmen ist. Da die Tatsachenfeststellung des BVerfG angesichts der notwendigen Abgrenzung zwischen Rechtsetzung und Rechtsanwendung jedenfalls von den normativen Grenzen der Ver-

27 BVerfGE 106, 62 (137).

28 Dazu näher C. Möllers, Gewaltengliederung. Legitimation und Dogmatik im nationalen und internationalen Vergleich, 2005, S. 95 ff., 105 ff. Zur Gebundenheit und Fallbezogenheit als Eigenschaften der Rechtsanwendung gegenüber der Rechtsetzung vgl. auch Hwang (Fn. 11), S. $129 \mathrm{ff} ., 212 \mathrm{ff}$.

29 Vgl. Möllers (Fn. 28), S. 105.

30 Dazu näher unten III.

31 So Möllers (Fn. 28), S. 95. 
fassung abhängen muss, liegt es nahe, dass sie schon aus methodologischen Gründen niemals grenzenlos sein kann. Vielmehr ist darauf hinzuweisen, dass die Einschätzungsspielräume des Gesetzgebers gerade aus den Grenzen der verfassungsgerichtlichen Rechtsanwendung erfolgen, die sich auf zweierlei zurückführen lassen, nämlich zum einen auf die Eigenschaft sowie die Funktionsgrenzen der Verfassungsnorm und zum anderen auf die Bedeutung und Begrenzung der verfassungsgerichtlichen Kompetenz zur Konkretisierung. Dadurch erklärt sich nicht nur, weshalb und wie der gesetzgeberische Einschätzungsspielraum zu begründen ist, sondern auch, welch große Bedeutung die methodologische Gegenüberstellung zwischen Rechtsanwendung und Rechtsetzung für die Bestimmung seiner Reichweite einnimmt.

\section{Tatsachenfeststellung im Rahmen verfassungsgerichtlicher Konkretisierung}

Wie dargelegt, hängt zwar die Inhaltsbestimmung der Verfassungsnorm gelegentlich von den generellen Tatsachen ab, deren Ermittlung auch gerade in diesem Sinne mit der Konkretisierungskompetenz des BVerfG zusammenhängt. Insofern ist Karl Engisch zuzustimmen, wenn er sagt: „Die Lebensordnung liefert dem Recht nicht nur den Gegenstand, sondern auch den Inhalt. "32 Dies bedeutet aber natürlich nicht, daß an dieser Stelle kein Unterschied zwischen Norm- und Tatsachenerkenntnissen besteht. Wie Carl Schmitt dargelegt hat: „Der Richter kann keinen Tatbestand aufnehmen, ohne dass ihm Gesetze, die Anwendung finden sollen, bereits , vorschweben' oder mehr oder weniger bewusst sind.... Der Tatbestand bedeutet daher bereits das Ergebnis einer Spezifikation, durch die ein neues Gebilde geschaffen ist und mit diesem Tatbestand allein hat es der Jurist zu tun. Die vollendete Geschlossenheit der Welt rechtlicher Normen ist damit gesichert. "33 Auf dieser Grundlage erklärt sich, dass die Verfassung nicht alle, sondern lediglich rechtserhebliche Tatsachen zum Gegenstand machen kann, ${ }^{34}$ die als Tatbestände der Verfassungsnorm gelten und sich infolgedessen durch das BVerfG konkretisieren lassen. Daher ist festzustellen, dass die Reichweite der rechtserheblichen bzw. von der Verfassung regulierten Tatsachen davon abhängt, wie ausführlich die Tatbestandmerkmale der einzelnen Verfassungsvorschriften vorgesehen sind. Je konkreter sich die die tatsächlichen Voraussetzungen zugrunde legenden verfassungsrechtlichen Tatbestände darstellen, desto umfangreichere Tatsachen kann die Verfassung regeln. So begriffen wird verdeutlicht, dass das BVerfG in der neueren Entscheidung zum Altenpflegegesetz die Neufassung des Art. 72 Abs. 2 GG deshalb als eine Vorschrift begriff, die justitiabel ist und dem Bundesgesetzgeber keinen Beurteilungsspielraum hinsichtlich ihrer Voraussetzungen überlässt, ${ }^{35}$ weil diese Neufassung die alte Bedürfnisklausel

32 K. Engisch, Die Idee der Konkretisierung in Recht und Rechtswissenschaft unserer Zeit, 2. Aufl. 1968, S. 97.

33 C. Schmitt, Der Wert des Staates und die Bedeutung des Einzelnen, 1917, S. 32.

34 Vgl. an dieser Stelle auch D. Grimm, Grundrechte und soziale Wirklichkeit. Zum Problem eines interdisziplinären Grundrechtsverständnisses, in: W. Hassemer (Hrsg.), Grundrechte und soziale Wirklichkeit, 1982, S. 39 ff., 44: „Darin liegt kein Rückfall hinter Kant und Kelsen, denn es wird nicht ein Sollen aus einem Sein abgeleitet, sondern lediglich festgestellt, dass sich ein Sollen nur angesichts eines Seins formulieren lässt und insoweit Seinselemente in sich aufnehmen muss.".

35 Vgl. BVerfGE 106, $62(135,142)$. 
durch eine relativ konkrete und voraussetzungsvolle Erforderlichkeitsklausel ersetzt hat. ${ }^{36}$

Während einerseits ausführliche verfassungsrechtliche Tatbestände mehr Tatsachen zum Gegenstand machen und insofern größeren Raum für die verfassungsgerichtliche Konkretisierung durch dessen Tatsachenermittlung eröffnen, muss andererseits gerade in dieser Hinsicht anerkannt werden, dass die Konkretisierung des BVerfG, die im Rahmen der Verfassungsrechtsanwendung vorzunehmen ist, auf die normative Reichweite der Verfassung zu beschränken ist. Dies hat zur Folge, dass die Konkretisierung sich erst nach den Vorgaben der Verfassung vollzieht und sich insofern nicht als Ausdehnungsmittel der Verfassungsgerichtsbarkeit ansehen lässt. Die allgemeine Ansicht, das Bedürfnis nach der verfassungsgerichtlichen Konkretisierung werde umso größer, je weniger die angewandte Verfassung aussage, muss in diesem Sinne vorsichtig überlegt und nur mit Vorbehalt angenommen werden. Solange die Konkretisierung als Rechtsanwendung gilt, ist die Tatsache sogar umgekehrt aufzufassen: Von der verfassungsgerichtlichen Konkretisierung ist desto geringeres zu erwarten, je weniger die angewandte Verfassung vorschreibt. ${ }^{37}$ Denn: Im Rahmen der Rechtsanwendung kann die Konkretisierung kein Vorgang sein, in dem das Gericht aus seiner Tatsachenermittlung die Normen selbst gestaltet. ${ }^{38}$ So verstanden bedeutet Konkretisierung nicht, wie Engisch ausdrückt, „Orientierung des Rechts an der Wirklichkeit“, ${ }^{39}$ wobei die Richter dazu berufen seien, das Sollen aus dem Sein abzuleiten, deren Stellung folglich ,keine andere sein kann als die des Gesetzgebers". ${ }^{40}$ Die neuhegelianische Auffassung, die zwischen Rechtsnorm und richterlicher Entscheidung ein dialektisches Verhältnis sieht und sich so formulieren lässt, ,nur in der Auslegung oder Interpretation konkretisiert

36 Vgl. BVerfGE 106, 62, $142 \mathrm{f}$.

37 Dies zeigt sich auch bei Struck (Fn. 25), S. 27, wo angedeutet wurde, dass die Konkretisierung eines Rechtsanwendungsorgans das Vorliegen konkreter Vorgaben des Rechtstextes voraussetzen muss: ,Je konkreter der Text, desto wichtiger wird es bei sozialem Wandel - also immer - gesetzgeberische Wertungen, Motivationen und deren faktische Voraussetzungen zu erkennen.".

38 Vgl. auch Grimm (Fn. 34), S. 47 f.

39 Engisch (Fn. 32), S. 96. Vgl. auch die kritische Bemerkung bei Bernd Rüthers: „Die Wirklichkeit bekommt eine Recht-setzende Funktion." B. Rüthers, Entartetes Recht. Rechtslehren und Kronjuristen im Dritten Reich, 2. Aufl. 1989, S. 65 (Hervorhebung im Original).

40 Engisch, ebenda, S. 120. Engisch behauptet: „Wo der Richter, freies Recht' schafft, wie bei der Lückenausfüllung, da mag er sich bei Auswahl der ,rechten Mittel zum rechten Zweck oder bei Aufdeckung und Bewertung der einschlägigen Interessen oder bei Orientierung an der Überlieferung und der ,bewährten Lehre' ebenso weit an den Realien ausrichten wie ein Gesetzgeber dies bei Findung des ,richtigen Rechts“ tun muss.“ Engisch, ebenda, S. 121. Dabei zeigt sich jedoch, dass die Gebundenheit des Richters gar ignoriert wird. 
das Recht sich zum Recht in seiner Wirklichkeit“, ${ }^{41}$ steht der traditionellen Haltung zur Rechtsanwendung entgegen und vernachlässigt, dass auch im Rahmen richterlicher Konkretisierung die Bindungskraft der (Verfassungs-)norm niemals preisgegeben werden darf, deren Grenzen infolgedessen anzuerkennen und nicht durch die an der Wirklichkeit orientierte verfassungsgerichtliche Konkretisierung zu überwinden sind. Die normativen Grenzen der Verfassung gelten gerade in dieser Hinsicht als die Grenzen der verfassungsgerichtlichen Konkretisierung.

Dem Vorhergehenden ist zu entnehmen, dass die Grenze der verfassungsgerichtlichen Überprüfung auf der einen Seite und die Reichweite der gesetzgeberischen Einschätzungsspielräume auf der anderen Seite auf die Konkretisierungsfähigkeit des BVerfG und letztendlich auf die Konkretisierungsfähigkeit der verfassungsrechtlichen Tatbestandmerkmale zurückgehen müssen. Genau an dieser Stelle versteht sich, weshalb die Ungewissheit über die empirischen Daten zur Einräumung der gesetzgeberischen Spielräume führen muss: Während gesicherte empirische Daten in dem Sinne rechtserheblich und konkretisierungsfähig sind, dass sie sich durch die verfassungsgerichtliche Konkretisierung in die verfassungsrechtlichen Tatbestände transformieren lassen, ist die empirische Unsicherheit im Hinblick auf deren Zukunftsbezogenheit nicht durch die verfassungsgerichtliche Konkretisierung in rechtserhebliches Material umzusetzen. Soweit also die zu ermittelnden Tatsachen empirisch unsicher erscheinen, muss auch in Rede stehen, ob und inwiefern die Verfassung materiell-rechtliche Maßstäbe für die Konkretisierung des BVerfG liefern kann, denn angesichts ihrer Offenheit und Unbestimmtheit können sich die Tatsachen als solche nicht durch die verfassungsgerichtliche Konkretisierung als rechtserhebliche Tatsachen bzw. als festgestellte verfassungsrechtliche Tatbestände darstellen. Daher zeigt sich deutlich, dass die empirische Unsicherheit über die normativen Vorgaben der Verfassung hinausgehen und in diesem Sinne keiner verfassungsgerichtlichen Kontrolle unterliegen kann, auch wenn die verfassungsrechtlichen Tatbestandmerkmale umfassend und ausführlich beschrieben werden. Gerade deswegen schließt das BVerfG trotz seiner Auslegung zum Art. 72 Abs. 2 GG n.F. die Einschätzungsspielräume des Gesetzgebers nicht aus. Vielmehr legt es dar: „Die Reichweite gerichtlicher Nachprüfung dieser Tatbestandsmerkmale hängt von ihrer Konkretisierungsfähigkeit und der Weite eines dem Bundesgesetzgeber einzuräumenden Ein-

41 So W. Schönfeld, Der Traum des positiven Rechts, AcP 135, S. 1 ff., 43, zitiert nach K. Larenz, Methodenlehre der Rechtswissenschaft, 6. Aufl. 1991, S. 109. Ähnlich F. Müller/R. Christensen, Juristische Methodik, 9. Aufl. Bd. I, 2004, Rn. 14. Infolgedessen führt Wolfgang Fikentscher aus: „Der Neuhegelianismus ist also die Quelle der Idee der Konkretisierung des Rechts, eine Haltung zur Rechtsanwendung, die neben der traditionellen Auffassung, das Gesetz werde auf den Fall angewandt, zur zweiten bedeutenden deutschen Tradition bis heute geworden ist." W. Fikentscher, Methoden des Rechts in vergleichender Darstellung, Bd. III: Mitteleuropäischer Rechtskreis, 1976, S. 299. Zum Neuhegelianismus vgl. ferner Larenz, ebenda, S. 102 ff.; O. Lepsius, Die gegensatzaufhebende Begriffsbildung: Methodenentwicklungen in der Weimarer Republik und ihr Verhältnis zur Ideologisierung der Rechtswissenschaft im Nationalsozialismus, 1994, S. 271 ff. Dennoch ist nicht zu verkennen, dass auch die Neuhegelianer das normorientierte Rechtsbewusstsein des Richters nicht außer Acht lassen (dazu näher Larenz, ebenda, S. 109). Dadurch unterscheidet sich das neuhegelianische SeinSollen-Verständnis immer noch vom US-amerkianischen. Dazu Lepsius (Fn. 2), S. 8: „In Deutschland stritt man um Werte, in den USA um Fakten. In Deutschland ging es um geistiges Sein und Idealität, in den USA um empirisches Sein und Realität.“. 
schätzungsspielraums bei der Ermittlung von Tatsachen und bei Prognosen tatsächlicher Entwicklungen ab. "42 So gesehen liegt es nahe, dass der Gesetzgeber in der Entscheidung zur Kampfhundeverordnung seine Einschätzungsspielräume zurück gewinnt, soweit die festzustellenden Tatsachen hier angesichts der fehlenden verfassungsrechtlichen Maßstäbe bei der empirischen Unsicherheit nicht in der Lage sind, sich völlig in das Konkretisierungsmaterial zu transformieren: „Dabei steht dem Gesetzgeber nicht nur bei der Festlegung der von ihm ins Auge gefassten Regelungsziele, sondern auch bei der Beurteilung dessen, was er zur Verwirklichung dieser Ziele für geeignet und erforderlich halten darf, ein weiter Einschätzungs- und Prognosespielraum zu, der... nur in begrenztem Umfang überprüft werden kann. Bei der Einschätzung von Gefahren, die der Allgemeinheit drohen, und bei der Beurteilung der Maßnahmen, die der Verhütung und Bewältigung dieser Gefahren dienen sollen, ist der Beurteilungsspielraum des Gesetzgebers erst überschritten, wenn die gesetzgeberischen Erwägungen so fehlsam sind, dass sie vernünftigerweise keine Grundlage für derartige Maßnahmen abgeben können. “43 Die Folge also: „Es ist Sache des Gesetzgebers, im Hinblick auf den jeweiligen Lebensbereich darüber zu entscheiden, ob, mit welchem Schutzniveau und auf welche Weise Situationen entgegengewirkt werden soll, die nach seiner Einschätzung zu Schäden führen können. “44 Beachtenswert ist dabei, dass gerade die empirische Unsicherheit, wobei die Verfassungsnorm keine materiell-rechtlichen Maßstäbe für das BVerfG vorgeben kann, die Konkretisierung des BVerfG hindert und infolgedessen die Einschätzungsspielräume des Gesetzgebers einräumen muss: „Es ist nicht vorhersehbar, unter welchen konkreten Umständen ein Hund dieser Rassen sich dem Einfluss des Halters entzieht und Menschen angreift. Im Hinblick auf das hohe Gewicht, das dem Schutz des menschlichen Lebens und der menschlichen Gesundheit in der Werteordnung des Grundgesetzes zukommt, und mit Rücksicht auf die schwerwiegenden Folgen, die Beißvorfälle unter Beteiligung von Hunden im Sinne des § 2 Abs. 1 Satz 1 HundVerbrEinfG wegen deren Stärke und Beißkraft für diese Schutzgüter haben können, bilden die genannten Daten vor diesem Hintergrund zusammen mit den oben wiedergegebenen Äußerungen des fachwissenschaftlichen Schrifttums eine ausreichende Grundlage für ein Handeln des Gesetzgebers, Vorkehrungen gegen den Eintritt von Schädigungen durch Hunde der erwähnten Rassen zu treffen." 45 Auch bereits im Mitbestimmungsurteil hat das Gericht darauf hingewiesen, inwieweit die empirische Unsicherheit hindernde Auswirkungen auf die Rechtsanwendungs- bzw. Konkretisierungsbefugnis des BVerfG hat: „Das Gesetz regelt einen Ausschnitt komplexer, schwer übersehbarer Zusammenhänge; diese hängen ihrerseits von Faktoren einer nicht auf die Bundesrepublik beschränkten Entwicklung ab, die sich zuverlässiger Einschätzung entziehen. Bei dieser Sachlage kann jedenfalls nicht gefordert werden, dass die Auswirkungen des Gesetzes mit hinreichender Wahrscheinlichkeit oder gar Sicherheit übersehbar sein müssten, zumal Rechtsgüter wie das des Lebens oder der Freiheit der Person nicht auf dem Spiele stehen. " ${ }^{66}$ Ebenfalls in diese Richtung weist das Gericht in der Entscheidung zur Arbeitnehmerüberlassung: „Bei der verfassungsgerichtlichen Nachprüfung ist besondere

42 BVerfGE 106, 62 (143).

43 BVerfGE 110, 141 (157f.).

44 BVerfGE 110, 141 (159).

45 BVerfGE 110, 141 (163).

46 BVerfGE 50, 290 (333). 
Zurückhaltung auch deswegen geboten, weil der Gesetzgeber bei der Wiederherstellung der durch illegale Leiharbeit gestörten Ordnung auf dem Teilarbeitsmarkt des Baugewerbes auf besonders komplexe, schwer überschaubare und im einzelnen unklare Verhältnisse einwirken muss. “47

III. Verfassungsgerichtliche Rechtsanwendung vs. gesetzgeberische Rechtsetzung vor dem Hintergrund empirischer Unsicherheit

Die vorangehende Darstellung spricht dafür, dass die empirische Unsicherheit die verfassungsgerichtliche Konkretisierung nicht begründet, sondern umgekehrt hindert, weil sie sich nicht durch die verfassungsgerichtliche Konkretisierung in rechtserhebliches Material bzw. in einen verfassungsrechtlichen Tatbestand umsetzen lässt. Das heißt auch, dass das BVerfG unter diesen Umständen nicht dazu befugt ist, die Verfassung anhand der ungewissen empirischen Tatsachen zu konkretisieren und dadurch die gesetzgeberischen Entscheidungen verfassungsrechtlich zu überprüfen. Der Vorstellung von Engisch, dass ,nicht das Gesetz auf einen Fall angewandt wird, um ihn zu lösen, sondern dass sich die Lösung einer Rechtsfrage aus einem Hin- und Herwenden des Blicks zwischen Gesetz und Fall, aus einer schrittweisen Konkretisierung allgemeiner Rechtssätze über immer mehr verfeinerte Normen bis hin in den wirklichen Sachverhalt ergibt" ${ }^{48}$ ist deshalb nicht zu folgen, weil sie die Eigenheiten der richterlichen Rechtsanwendung vernachlässigt, die sich nicht nur aus der Einzelfallbezogenheit, sondern vielmehr auch aus der Gebundenheit der Rechtsprechung ergeben müssen. Die Notwendigkeit einer Konkretisierung durch das BVerfG entsteht zwar aus der Unvollkommenheit der Verfassungsnorm; allerdings kann sie angesichts der methodologischen Gegenüberstellung zwischen Rechtsetzung und Rechtsanwendung keineswegs darauf abzielen, die Grenzen der Verfassungsnorm durch die Ineinssetzung von Sein und Sollen zu überschreiten. Konkretisierung stellt sich, wie Hans Kelsen darlegte, als ein Prozess stetig zunehmender Individualisierung ${ }^{49}$ dar, die sich im Rahmen der Verfassung bewegt und gerade insofern die normativen Schranken der Verfassung nicht überschreiten darf, auch wenn sie eine Tatsachenermittlung zugrunde legen muss. Zwar kann sich das schöpferische Element einer richterlichen Konkretisierung in die (verfassungsgericht-

47 BVerfGE 77, 84 (106f.).

48 So die Darlegung bei Fikentscher (Fn. 41), S. 299. Ähnlicherweise versteht Larenz die richterliche Rechtsanwendung/Konkretisierung als ein dialektisches, den Gegensatz von Recht und Wirklichkeit überwindendes Verfahren. Vgl. z.B. K. Larenz, Deutsche Rechtserneuerung und Rechtsphilosophie, 1934, S. 32: „Indem der Richter diese Rückbeziehung der Norm auf die konkrete Rechtsidee und damit auf das lebendige Rechtsbewusstsein der Gemeinschaft vollzieht, trägt er nichts Fremdes an die Norm heran, sondern löst sie aus der Erstarrung, der sie im positivistischen Denken anheimfällt, und holt damit erst das lebende Recht aus ihr heraus.".

49 Vgl. H. Kelsen, Reine Rechtslehre. Mit einem Anhang: Das Problem der Gerechtigkeit, 2. Aufl. (1960), Nachdruck 2000, S. 242. 
liche) Bewertung von belegten Tatsachen einfließen lassen; ${ }^{50}$ allerdings ist daran festzuhalten, dass es im Falle empirischer Ungewissheit nicht in der Lage ist, die Kluft von faktischem Sein und normativem Sollen zu überbrücken. Gerade an dieser Stelle verdeutlichen sich die Unterschiede zwischen der Rolle des rechtsanwendenden Verfassungsgerichts und der des rechtsetzenden Gesetzgebers: Wegen der in der kontinentaleuropäischen Rechtstradition unentbehrlichen Distanz zwischen Normativität und Faktizität ${ }^{51}$ sind die Richter als typische Juristen dazu berufen, die festgestellten Tatsachen am Maßstab der rechtlichen Vorgaben normativ zu bewerten; ${ }^{52}$ allerdings haben sie gleichzeitig an der normativen Verschlossenheit festzuhalten, die auf eine Bewertung oder Einschätzung empirischer Unsicherheit verzichten muss. Demgegenüber bemüht sich der Gesetzgeber um die zukunftsorientierte politische Gestaltung, ${ }^{53}$ die gerade nicht von normativen, sondern umgekehrt von faktischen Voraussetzungen ausgehen muss. Infolgedessen muss einerseits die gesetzgeberische Bewertung von belegten Tatsachen im Rahmen des Grundgesetzes der verfassungsgerichtlichen normativen Überprüfung unterliegen; andererseits aber ist sie im wesentlichen nicht auf die normative Geschlossenheit des Rechtssystems beschränkt. Die Folge also: Während die empirische Unsicherheit die Konkretisierung durch das BVerfG versperrt, hindert sie den Gesetzgeber nicht daran, die in Rede stehenden Interessen zu erwägen und dadurch Recht zu setzen, denn der Gesetzgeber hat gerade die Aufgabe, eine Entscheidung nicht aus der vorgegebenen Norm, sondern aus der Wirklichkeit und auch für die Wirklichkeit zu treffen, die sich entweder auf gesicherte Tatsachen oder auf ungewisse Zukunft beziehen kann. Gerade auf dieser Grundlage überzeugt Peter Lerches Unterscheidung zwischen der Verfassungskonkretisierung durch das Verfassungsgericht auf der einen Seite und durch den Gesetzgeber auf der anderen Seite: „In Fällen dieser Art, also gesetzgeberischer Inhaltsfüllung, wird im Grunde die Verfassung nicht nur ,konkretisiert'. Es wird nicht ein schon Vorgegebenes konkreter gemacht, fixierend ausgestaltet; vielmehr wird die Substanz selbst erst geschaffen, wenn auch in verfassungsrechtlichen Grenzen. Nicht nur Konkretisierung, sondern Konstituierung, Erzeugung der Substanz selbst, ist hier Befugnis und Verantwortung des Gesetzgebers. "54 Somit zeigt sich nicht nur, wodurch die Rechtsetzungskompetenz des Gesetzgebers gegenüber der Rechtsanwendung des Verfassungsgerichts gekennzeichnet ist, sondern auch, woraus die Einschätzungsprärogative des Gesetzgebers entsteht und entstehen soll. Eben angesichts der Grenzen der Verfassungsnorm und folglich der der verfassungsgerichtlichen Konkretisierung muss das BVerfG dem Gesetzgeber einen Einschätzungsspielraum einräumen, inner-

50 Dazu Böckenförde, Grundrechte als Grundsatznormen (Fn. 6), S. 22 (Fn. 85) unter Hinweis auf $H$. Huber, Die Bedeutung der Grundrechte für die sozialen Beziehungen unter den Rechtsgenossen, abgedruckt in: E. Forsthoff (Hrsg.), Rechtsstaatlichkeit und Sozialstaatlichkeit, 1968, S. $259 \mathrm{ff} ., 284 \mathrm{f}$.

51 Hinter dem Common Law verbirgt sich jedoch eine andere Sein-Sollen-Ausrichtung. Dazu O. Lepsius, Verwaltungsrecht unter dem Common Law: amerikanische Entwicklungen bis zum New Deal, 1997, S. 245 ff.; ders. (Fn. 2), S. 4 ff.

52 Eben darin besteht die eigenartige Bedeutung der Rechtswissenschaft. Vgl. dazu Grimm (Fn. 34), S. 47 f.; Lepsius (Fn. 2), S. 11-13.

53 Dazu näher Badura (Fn. 19), S. 542; Ossenbühl (Fn. 2), S. 501 f.

54 P. Lerche, Facetten der „Konkretisierung“ von Verfassungsrecht, in: ders. (Fn. 22), S. 86 ff., 103. Dort hat Lerche offensichtlich einen relativ lockeren bzw. extensiven Begriff der Konkretisierung angenommen. 
halb dessen der Gesetzgeber die Befugnis hat, aufgrund ungewisser Tatsachen eine Regelung zu erlassen. So gesehen kann die verfassungsgerichtliche Überprüfung einer gesetzgeberischen Prognose zu weit gehen, indem sie sich nicht nur auf das Verfahren sowie das Ergebnis der Prognose ${ }^{55}$ sondern darüber hinaus auf den Regelungsinhalt anhand der Prognose erstreckt. Insoweit ist der abweichenden Meinung der Richterinnen Osterloh und Lübbe-Wolff und des Richters Gerhardt zum Urteil des Zweiten Senats vom 27. Juli 2004 zu folgen, als sie diese Entscheidung so kritisierte, dass „das BVerfG bei der Prüfung der Bundeskompetenz zur Entscheidung über Sachfragen politischer Natur genötigt wird, für deren Beurteilung keine verfassungsrechtlichen Maßstäbe vorhanden sind. "56 Da die im Art. 72 Abs. 2 GG vorgegebenen Tatbestandmerkmale lediglich die Regelungsvoraussetzungen des Bundesgesetzgebers umfassen, darf sich die Tatsachenermittlung des BVerfG nicht auf den bundesgesetzgeberischen Regelungsinhalt erstrecken, dem eine zukunftsorientierte, auf Ungewissheit basierende und infolgedessen nicht in verfassungsrechtliche Tatbestände umzusetzende und auch nicht am Maßstab der Verfassung zu überprüfende Beurteilung zugrunde liegt. ${ }^{57}$ Die Einschätzungsprärogative des Gesetzgebers hat das BVerfG gerade deshalb zu beachten, weil sie das Vorliegen von empirischer Unsicherheit voraussetzt, die die verfassungsgerichtliche Rechtsanwendung, nicht aber die gesetzgeberische Rechtsetzung blockiert.

55 Vgl. dazu BVerfGE 106, 62 (152 f.); 111, 226 (255): „Der Prognose müssen Sachverhaltsannahmen zu Grunde liegen, die sorgfältig ermittelt sind oder sich jedenfalls im Rahmen der gerichtlichen Prüfung bestätigen lassen. Die Prognose muss sich methodisch auf ein angemessenes Prognoseverfahren stützen lassen, und dieses muss konsequent verfolgt worden sein. Das Prognoseergebnis ist daraufhin zu kontrollieren, ob die die prognostische Einschätzung tragenden Gesichtspunkte mit hinreichender Deutlichkeit offen gelegt worden sind oder ihre Offenlegung jedenfalls im Normenkontrollverfahren möglich ist und ob in die Prognose keine sachfremden Erwägungen eingeflossen sind." An dieser Stelle zeigt sich wiederum deutlich, dass das BVerfG um so konkretisierungs- und überprüfungsfähiger wird, je ausführlicher die verfassungsrechtlichen Tatbestandmerkmale sich darstellen. Auf der anderen Seite ist jedoch nicht zu vernachlässigen, dass, wie schon dargelegt, auch im Rahmen der Voraussetzungen des Art. 72 Abs. 2 GG die empirische Ungewissheit über die verfassungsrechtlichen Tatbestände hinausgehen und die Konkretisierungsfähigkeit des BVerfG hindern kann.

56 BVerfGE 111, 226, $274 \mathrm{ff}$. (278).

57 Deshalb fuhr die abweichende Meinung fort: „Indem die Senatsmehrheit über die Prüfung der Erforderlichkeit des Zugriffs des Bundesgesetzgebers auf einen bestimmten Regelungsgegenstand (hier: Voraussetzungen des Zugangs zum Professorenberuf) hinaus nun auch die Erforderlichkeit des jeweiligen Regelungsinhalts (hier: Juniorprofessur statt Habilitation) zum Gegenstand der Kompetenzprüfung nach Art. 72 Abs. 2 GG macht, verschiebt sie die materiellrechtliche Erforderlichkeitsprüfung auf die Kompetenzebene. Damit wird auch die inhaltliche Gesetzesgestaltung an die in Art. 72 Abs. 2 GG genannten Zielgrößen der Erforderlichkeit gebunden, dem Gesetzgeber also die Freiheit der politischen Zielwahl genommen. Zudem vorverlagert die Senatsmehrheit die inhaltsbezogene Erforderlichkeitsprüfung damit auf ein Feld, auf dem nach der Entscheidung des Senats zum Altenpflegegesetz ein Beurteilungsspielraum des Gesetzgebers nicht anzuerkennen ist.“ (S. 279). 


\section{Folgen: Die Einschätzungsspielräume des Gesetzgebers aus arbeitsteiligen Erwägungen}

Während gesicherte Tatsachen sich zum Gegenstand und gleichzeitig auch zum Inhalt der Verfassung machen und gerade in diesem Sinne als rechtserhebliche Tatsachen die Konkretisierung des BVerfG ermöglichen, wobei die verfassungsgerichtliche Bewertung unumgänglich wird und insofern den Einschätzungsspielraum des Gesetzgebers ausschließt, ${ }^{58}$ führt hingegen die empirische Unsicherheit im Rahmen der Tatsachenermittlung folgerichtig zu einer Einschätzungsprärogative des Gesetzgebers, dessen Rechtsetzungskompetenz im wesentlichen zukunftsbezogen und infolgedessen darauf verpflichtet ist, empirische Ungewissheit durch politische Beurteilung und Gestaltung zu überwinden. So gesehen lassen sich die Einschätzungsspielräume des Gesetzgebers nicht durch das Demokratieprinzip, sondern erst durch die methodologische Trennung zwischen gesetzgeberischer Rechtsetzung und verfassungsgerichtlicher Rechtsanwendung begründen, die die normativen Vorgaben der Verfassung voraussetzen und deswegen die verfassungsrechtlichen normativen Grenzen in sich aufnehmen muss, außerhalb denen der Gesetzgeber seinen Ort findet.

Aus dieser Perspektive ist weiterhin festzustellen, dass die hier vertretenen arbeitsteiligen Erwägungen, die gerade von der Gegenüberstellung zwischen Sein und Sollen, zwischen Faktizität und Normativität und zwischen Rechtsetzung und Rechtsanwendung ausgehen, nicht nur für die Herausstellung der Eigenschaft sowie der Grenze der Rechtswissenschaft gegenüber ihren Nachbarwissenschaften, ${ }^{59}$ sondern darüber hinaus für die Erklärung der Gewaltengliederung zwischen Verfassungsgerichtsbarkeit und Gesetzgebung gelten. Die Abstufung unterschiedlicher Kontrolldichte hängt in dieser Hinsicht gerade von der Reichweite der normativen Maßstäbe individueller Verfassungsvorschriften ab. Alle ins Spiel kommenden Kriterien - sowohl die Eigenart des in Rede stehenden Sachbereichs als auch die Bedeutung der auf dem Spiele stehenden Rechtsgüter - sind im Rahmen der methodologischen Gegenüberstellung von Rechtsetzung und Rechtsanwendung zu begreifen. So betrachtet soll einerseits die verfassungsgerichtliche Bewertung von belegten und sich deswegen in verfassungsrechtliche Tatbestände umsetzenden Tatsachen, die als juristische Aufgabe gilt, nicht aus kompetenzrechtlichen Überlegungen bzw. aus den sog. demokratischen Gründen vorgeworfen werden. Andererseits aber darf sie ohne verfassungsrechtliche Vorgaben nicht eintreten, die die ungewissen empirischen Daten nicht zum Gegenstand machen und sie in diesem Sinne freilassen müssen. Gerade auf der Grundlage, dass die Verfassung als Rechtsnorm nur begrenzte Wirklichkeit durch die rechtsanwendende verfassungsgerichtliche Kontrolle regeln kann und will, rechtfertigen sich die Einschätzungsspielräume des Gesetzgebers.

58 Vgl. dazu BVerfGE 106, 62 (151): „Soweit hingegen Unsicherheiten der Prognose durch gesicherte empirische Daten und verlässliche Erfahrungssätze ausgeräumt werden können, scheidet ein Prognosespielraum aus.".

59 Zur Grenzziehung zwischen Rechts- und Sozialwissenschaft durch eine auf der Sein-SollenDichotomie beruhende arbeitsteilige Erwägung vgl. Lepsius (Fn. 2), S. 3 ff. 